\title{
Oral Manifestations of Some Rare Diseases: A Narrative Review of the Radiological Findings in Orthopantomography
}

\author{
Luca Viganò $^{{ }^{*}}$, Cinzia Casu ${ }^{2}$, Mariapia Brignoglio ${ }^{3}$, Veronica Caria ${ }^{3}$, Marina Paola Lazzari ${ }^{4}$, Clarita Pellegrini ${ }^{3}$
}

\author{
${ }^{1}$ Department of Radiology, San Paolo Dental Building, University of Milan, Italy \\ ${ }^{2}$ Private Dental Practice, Cagliari, Italy \\ ${ }^{3}$ San Paolo Dental Building, University of Milan, Italy \\ ${ }^{4}$ IRCCS Ca' Granda Foundation General Hospital University of Milan, Italy
}

DOI: $10.36347 /$ sjds.2020.v07i10.003

| Received: 28.09.2020 | Accepted: 08.10.2020 | Published: 15.10.2020

*Corresponding author: Luca Viganò

Abstract

Original Research Article

Rare diseases have a prevalence that is not more than 5 cases per 10,000 people. Some rare diseases have oral manifestations. Among these conditions, there are some syndromes, such as Gorlin Goltz, Turner syndrome, SAPHO, Williams syndrome, Schimke Immuno, Cherubism, and Neurofibromatosis type 1. Considering their low incidence and high complexity, these disorders require a diagnostic and therapeutic approach based on the interaction of several specialists. In these field, the dentist plays a leading role: he is at the forefront of the early detection of diseases whose prodromal signs may forerun systemic manifestations by few years. Orthopantomography (OPT) allows to identify changes in bone structures and dental anomalies of shape and number, often associated with the above listed manifestations. The aim of this article is to show how the dentist, basing on some radiological evidences, may be able to make an early diagnosis or to formulate a diagnostic hypothesis that will need to be approved by a multidisciplinary team.

Keywords: Orthopantomography, panoramic radiograph, dental abnormalities.

Copyright $\left({ }_{0} 2020\right.$ The Author(s): This is an open-access article distributed under the terms of the Creative Commons Attribution 4.0 International License (CC BY-NC 4.0) which permits unrestricted use, distribution, and reproduction in any medium for non-commercial use provided the original author and source are credited.

\section{INTRODUCTION}

Rare diseases, as reported by the Istituto Superiore di Sanità (ISS), have a prevalence of 5 cases or less per 10,000 people.

They represent a topic of increasing interest for the healthcare system, due to the high number of patients affected in the whole world. The estimated total amount of rare diseases is approximately 7,000, and the $50-70 \%$ of them affects children.

About the $80 \%$ has a genetic cause, whereas the remaining $20 \%$ is a multifactorial condition resulting from the interaction between genetic and environmental factors. The onset is highly variable, and runs from childhood to adulthood.

Although their heterogeneity, rare diseases are characterized by several shared aspects, such as the difficulty in obtaining a fitting and prompt diagnosis, the lack of definitive therapies, the disease's evolution (often chronic and disabling), and the significant individual, familiar and social weight.
Some rare diseases have oral manifestations These include Gorlin Goltz syndrome, Turner syndrome, SAPHO, Williams syndrome, SchimkeImmuno, Cherubism and Neurofibromatosis type 1 .

These conditions, due to their low incidence and high complexity, require a diagnostic and therapeutic approach based on the interaction of several specialists.

The dentist can give a considerable and sometimes decisive contribution to the detection of the disease. As a matter of fact, although the concerning literature is quite restricted, as well as the majority of the results, which occasionally are in part contradictory, there are radiological findings that may be correlated to some rare diseases.

In the dental field a valid diagnostic support is represented by the orthopantomography (OPT). More specifically, it can be described as an extraoral radiographic technique which provides a panoramic image of teeth and bone supports. 
The aim of this article is to show how the dentist, basing on some radiological evidences, may be able to make an early diagnosis or to formulate a diagnostic hypothesis that will need to be approved by a multidisciplinary team.

\section{MATERIALS AND METHODS}

Pubmed, Google Scholar, and Scopus databases were searched for the name of each syndrome combined with keywords, such as "panoramic radiograph", "orthopantomography", "oral manifestations", "temporomandibular joint", and "dental abnormalities".

\section{Inclusion criteria were:}

- Studies on humans

- Studies in English
- Free full text

- Case report

- Review

- Systematic review

- Dental journal

- Articles published between 2010 and 2020

\section{Exclusion criteria were:}

- Articles published before 1987

- No free full text

In the beginning, 8940 articles resulted from the research on databases. After the application of the inclusion and exclusion criteria, a total of 36 articles was analyzed for this narrative review.

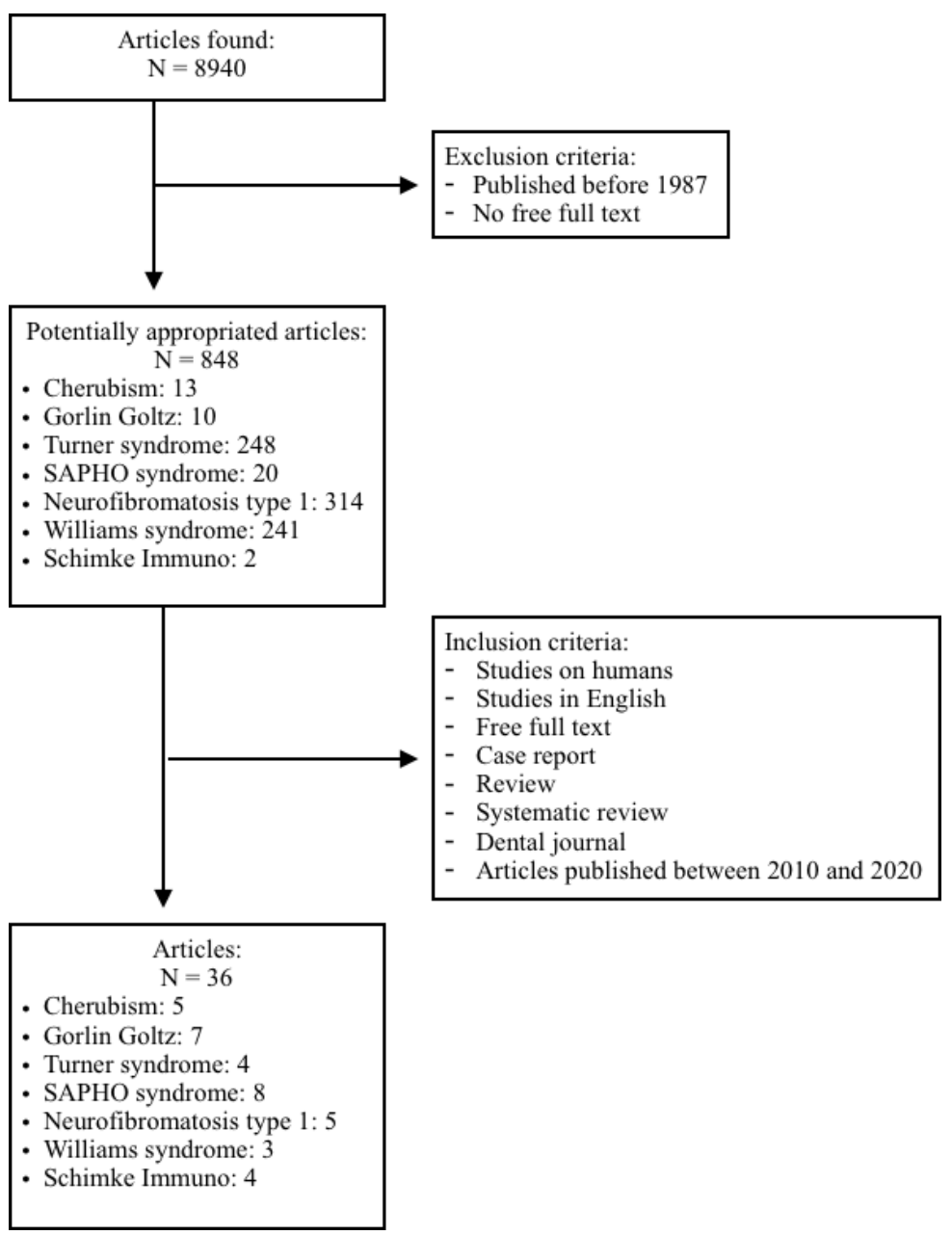

\section{DISCUSSION}

CHERUBISM: Cherubism, first described in 1933 by William Jones, is a dominant autosomal disease in which normal bone is replaced by cellular fibrous tissue and immature bone [1]. It is characterized by bilateral expansion of the jaw or jaw and mandible, although normally the mandible is more affected than the jaw. Patients are normal at birth and most of them develop the more or less symmetrical jaw and mandible expansion of entities ranging from 2 to 5 years [2]. The increase in bone volume produces a characteristic facial deformity consisting of a fullness of the cheeks and jaws with a typical plump face. Hence the name of the disease precisely because of the particular shape that assumes the face of the patient, that is rounded, with upward displacement of the orbits and exposure of the lower part of the scleres due to jaw injuries, so that the eyes appear facing the sky reminiscent of the 
appearance of the cherubs depicted in the Renaissance paintings [2]. The chubby appearance is accentuated by the swelling of the submandibular space due to lymphadenopathy that occurs in the early stages of the disease. It is also called "family fibrous dysplasia of the jaws", but a recent genetic research has shown that, considering the molecular level, it is a separate entity [3]. The main gene associated with cherubism is SH3BP2 which has been mapped to the $4 \mathrm{p} 16.3$ chromosome. Mutations in the $\mathrm{SH} 3 \mathrm{BP} 2$ gene influence bone remodeling. In fact, normal bone architecture is replaced by the proliferation of fibrous tissue, including a large number of osteoclasts, and the resulting bone septi give the characteristic multilocular appearance on X-rays [1-3]. This mutation induces an increase in osteoclastic activity with increased bone destruction. Mutations in the SH3BP2 gene also cause dysregulation of the Msx-1 gene, which is involved in regulating mesenchymal interaction in craniofacial morphogenesis. There is an increase in bone activity due to the up-regulation of Msx-1 which then stops at the end of molar development, leading to the remineralization of lesions $[4,5]$. Initially the disease was considered exclusively familiar, however sporadic cases were also reported. These can result from incomplete penetration or new mutations. So the absence of a positive familiar history does not exclude the possibility of cherubism $[2,5]$. About 300 cases of different ethnicities have been reported in the literature and it would appear that males are affected at roughly twice the frequency of females, although Papadaki et $a l$, believe that there is no gender difference [3, 5]. Considering their clinical behavior, lesions can be classified as aggressive, non-aggressive or quiescent, and each type corresponds to a specific age group (early childhood, adolescence, adulthood). In fact, the lesions increase in volume until the age of about 7 years, then stabilize, and when the patient goes from puberty to adulthood they begin to regress through bone remodeling, with progressive reduction of facial deformity [3, 5]. The classification system describes three degrees based on the anatomical position and extent of bone abnormalities: in Grade 1 lesions are confined to the lower molar region and coronoid processes. In Grade 2, in addition to the lesions of the first grade, other lesions appear in the jaw or mandible. In Grade 3 jaw and mandible are affected in a widespread way [1].

Through the study with panoramic X-ray, clearly defined multilocular radiotransparencies, with expansion and thinning of the bone corticals, can be highlighted. The mandible lesions appear to begin near the mandible angle and then spread to involve the body and branch, sparing the condyle as reported by Justyna Wagel et al., in a case report of a 5-year-old and by Silva et al., in the study of 8 clinical cases [2, 4]. During the progression of cherubism, the mandibular canal is moved without being damaged [2]. The lesions have a variable shape, are usually asymptomatic, and divided by thin bone trabecoles. In the jaw, the lesions begin in the region of the jaw tuberosity [3]. During puberty the lesions change from radiotransparents to radiopache, due to the process of reossification that implies the regression of pathology. Cherubism is also accompanied by abnormal dental rash as well as agenesis and the presence of ectopic or retained $t$ eeth. Teeth abnormalities include premature loss of deciduous teeth and displacement, absence of rash, developmental defect or agenesis of permanent teeth, especially of the second and third molar, and reabsorption of dental roots. Ectopic teeth are often involved in lesions. Malocclusion is common [2, 3].

Clinical signs vary: from subclinical cases to severe expansion associated with visual, respiratory, language-related, chewing and swallowing complications. In severe cases, fibro-bone lesions can invade the orbital walls causing the retraction of the lower eyelid, the dislocation of the globe, proptosis or double vision [2].

Respiratory complications are rare, but they may include sleep apnea, upper airway obstruction, and nasal airways obliteration [5]. Diagnosis is based on clinical signs, patients' age, familiar history and X-rays, and it can be confirmed by molecular analyses. Histological examination has a limited meaning for the diagnosis, showing abundant multinucleated giant cells scattered in a stroma of vascularized fibrous connective tissue [2, 5]. Hence we can say that the histological aspect is indistinguishable from that of other gigantocellular lesions of the bone, so that these pathological conditions cannot be distinguished on the basis of histology alone. Then, genotypic characterization is used to confirm the diagnosis.

There are differential diagnoses that should be taken into account in patients with suspected cherubism due to multilocular radio transparency in the jaws. One of these conditions is Gorlin-Goltz syndrome. Dental keratocystic tumors of this syndrome can be bilaterally found in both the jaw and mandible; they can be multilocular and, therefore, very similar to cherubism. However, such abnormalities are often less expansive than those present in cherubism; moreover, they usually are solid in cherubism and cystic in Gorlin-Goltz syndrome [1].

Hence, in case of suspected cherubism, radiographic examination is essential since the clinical presentation and the location and distribution of lesions can define the diagnosis. Then genetic testing is used for the final diagnosis. Clinical and radiological monitoring in the growth phase of lesions is recommended and the patient should be followed for any teething problems. Since the disease is usually selflimiting, surgery is not always indicated. However, surgery may be necessary in patients who have language impairment, chewing or swallowing and eye 
disorders, or for aesthetic reasons and to improve their quality of life [2, 4]. The psychological implications associated with disfigurement during childhood and adolescence should also be carefully assessed. The panoramic exam should also be the first choice at follow-up [1, 4]. Despite the possible severity of the signs and symptoms, the disease is benign and the prognosis is good; deformities can persist only in rare cases [5].

In conclusion, we can say that the knowledge of the clinical and radiographic alterations observed in patients with cherubism is important since the dentist may be the first professional to diagnose this disease [3].

\section{GORLIN GOLTZ}

Gorlin Goltz syndrome [6], first studied by Jarisch and White, is an autosomal dominant genetic condition with a high penetration rate and variable expression.

The incidence is 1 in 60,000 cases. Men and women are equally affected. Although the syndrome can affect all ethnic groups, Africans, Americans and Asians represent only 5\% of cases [7].

The main clinical features related to the syndrome are: the presence of multiple odontogenic keratocystic tumors $(75 \%)$, basal cell nevus carcinomas, skeletal, ophthalmic and neurological abnormalities.

The syndrome is caused by mutations of the PTCH 1 gene [8], on chromosome 9q, which codes for a transmembrane receptor that recognizes proteins of the SHH (sonic hedgehog) family, fundamental in the regulation of vertebrate organogenesis, such as in the differentiation of the fingers, limbs of the nervous system and teeth.

Typical signs of the syndrome emerge in the first, second or third decade. Multiple basal cell nevus carcinomas are the distinctive feature of the syndrome and they manifest as classic translucent papules or telangiectasias. Superficial lesions on palms of hands and feet occur in $75-90 \%$ of patients and in $50 \%$ we see epidermal cysts. Pain, swelling and inflammation of the maxillary cysts (odontogenic keratocysts) can be the most evident symptoms and usually occur around 13 years of age, together with dental agenesis.

In some rare cases there has been the malignant transformation of the maxillary cysts, or the presence of medulloblastoma, the second most common malignant tumor linked to the syndrome, which occurs around two years and has a frequency of 5\%. Some patients may experience seizures, mental retardation and other neurological abnormalities.
Musculoskeletal anomalies are also very common in $60-75 \%$ of cases and are often congenital, we can have spina bifida, frontal protrusion, third class prognathism, cleft palate, pectus excavatum, hypoplastic thumbs and other variants. Among the ophthalmic anomalies, on the other hand, we find congenital blindness, cataracts, strabismus and pathologies of the retina and iris.

Also, some patients during general anesthesia may be affected by a profound bradycardia. Diagnosis occurs through the recognition of some criteria, divided into major and minor.[9] Specifically, a diagnosis of Gorlin Goltz syndrome is made if we are in the presence of two major criteria or one major and two minor.

Major major criteria include

a) Multiple basal cell carcinomas (more than two) or basal cell carcinoma before age 20 ,

b) Odontogenic keratocysts of the jaw seen on histological examination,

c) Palmar or plantar vesicular lesions,

d) Bilamellar calcification of the scythe cerebral,

e) Bifid or fused ribs

f) Basal cell nevus syndrome.

Among the minor criteria, instead we have

a) Medulloblastoma,

b) Increase in head circumference,

c) Congenital, skeletal, radiological malformations (hemivertebrae, fusion of vertebral bodies and modeling defects of hands and feet),

d) Ovarian and cardiac fibroids.

In the event that it is still not possible to diagnose the syndrome through these criteria, a genetic test for Pitch 1 is used which confirms the diagnosis in patients without sufficient clinical diagnostic criteria, in patients with a member of the family concerned but who do not have criteria or before birth if there is a known family mutation.

According to MacDonalds-Jankowski [10], odontogenic keratocysts are generally located in the molar region of the jaw, are asymptomatic and can later cause swelling of the face.

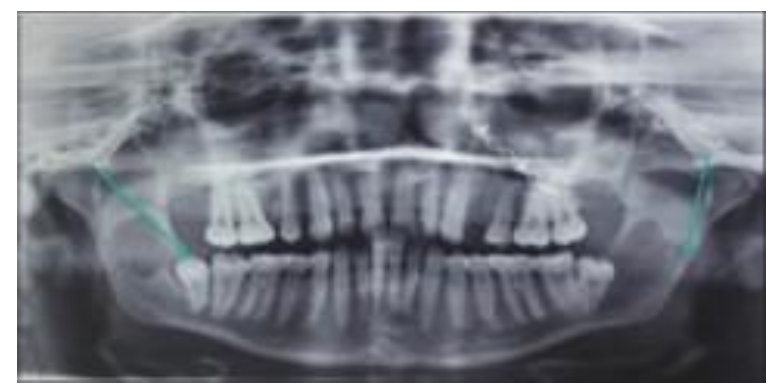

Fig-1: The orthopantomogram (OPT) shows two radiolucencies in the mandible (Courtesy of Gorlin - Goltz syndrome: An often missed diagnosis Ninan Thomas et al., [11] 


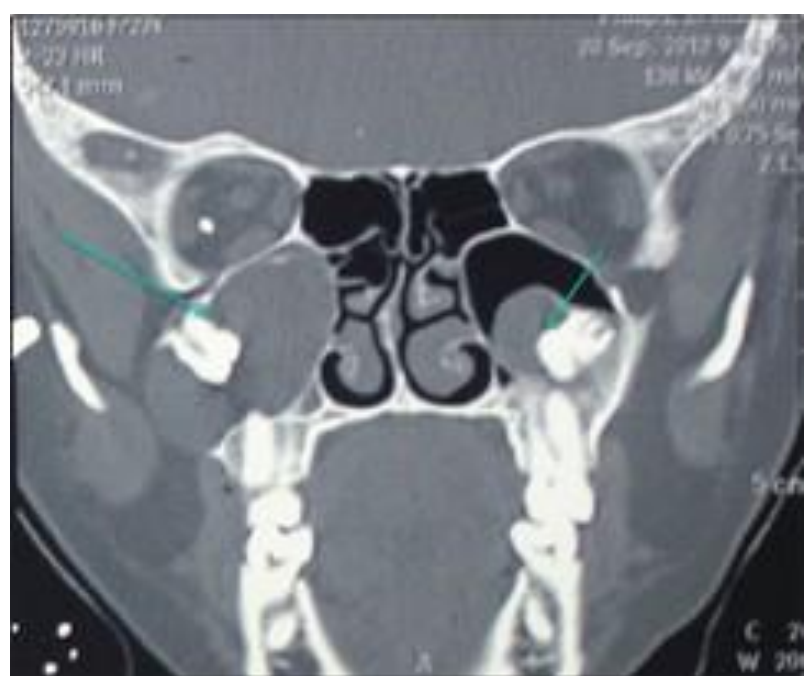

Fig-2: (CT) showed previous teeth lesions indicated by arrows (Courtesy of Gorlin - Goltz syndrome: An often missed diagnosis Ninan Thomas et al., [11]

Most of the criteria that can be found can be investigated by means of an orthopantomography commonly used in orthodontic treatment and in the dental clinic. In this syndrome on radiographic examination, single or multilocular radiolucencies in

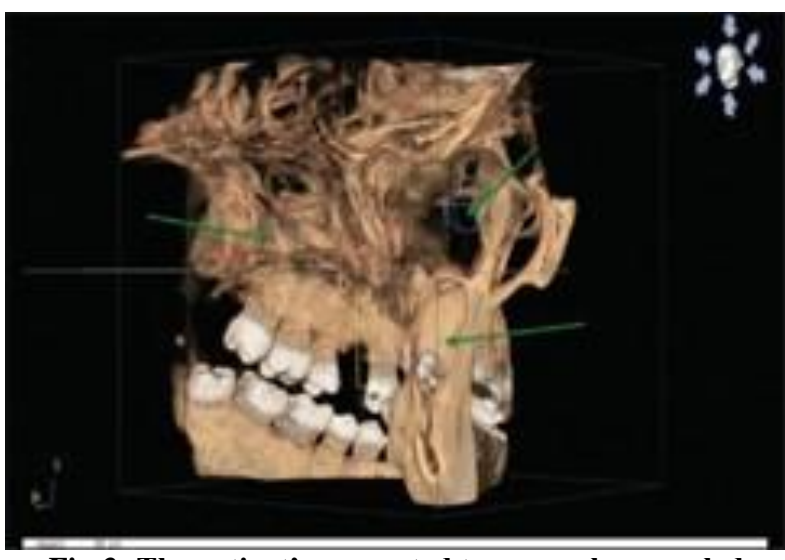

Fig-3: The patient's computed tomography revealed maxillary lesions extending almost half of the maxillary sinus with penetration of the posterior antral wall to the right (courtesy of Gorlin - Goltz syndrome: An often missed diagnosis Ninan Thomas et al., [11].

the body, in the corner or in the branch of the jaw, attributable to odontogenic cysts are noted.

Diagnostic criteria of nevoid basal cell carcinoma syndrome

\begin{tabular}{|l|l|}
\hline MAJOR & MINOR \\
\hline A) Basal cell carcinoma in patient $<20$ ys, or more than 2 & A) Medulloblastoma \\
\hline B) Odontogenic keratocyst of the jaw proven histologically & B) Macrocephaly \\
\hline C) Three or more palmar or plantal vesicular lesions & $\begin{array}{l}\text { C) One of the several congenital, skeletal } \\
\text { malformations (hemivertebrae, fusion of vertebral } \\
\text { bodies and modeling defects of hands and feet), }\end{array}$ \\
\hline D) Bilamellar calcification of the scythe cerebral & D) Ovarian and cardiac fibroids \\
\hline E) Bifid, fused or markedly splayed ribs & \\
\hline F) Basal cell nevus syndrome & \\
\hline
\end{tabular}

In children and adolescents, the cyst can cause damage in the development of the teeth, dislocation and delays in the eruption.

The recognition of odontogenic cysts is very important because they are often the first clinical manifestation of the syndrome and appear in the first decade, therefore earlier than the other diagnostic criteria, such as the presence of basal cell carcinoma or skeletal anomalies, which if present lead to a wellprognosis worst.

In a case report by Lazaridou et al., 2011, [12] is highlighted the rare case of a patient diagnosed with Gorlin Goltz syndrome following the detection of odontogenic keratocysts and other minor manifestations. After some time the malignant transformation of an odontogenic cyst into basal cell carcinoma, not at all common according to Ward and Cohen, has been highlighted by histological findings due to several factors such as: 1 the presence of a cyst that is involved in a malignant tumor which arises in an adjacent anatomical region and extends to the bone. 2 . The presence of a malignant tumor that undergoes a cystic transformation, or 3 . The existence of a cyst that undergoes a malignant transformation. This case demonstrates the importance of follow-up and careful histological investigation.

In conclusion, it is clear how much the dentist plays a key role in the early diagnosis of the disease and in the decrease in mortality associated with the syndrome. Patients with Gorlin Goltz syndrome need multidisciplinary management which includes surveillance by the dermatologist, in order to avoid metastasis of basal cell carcinoma, many screening especially at an early age. Magnetic brain resonances and repeated visits by the dentist to keep odontogenic cysts at the jaw level under control and prevent them from degenerating. Spine radiographs, cardiac and pelvic ultrasounds should also be performed in girls after menarche, as well as repeated hearing and vision visits over time. 


\section{TURNER SYNDROME}

Turner syndrome is a genetic disorder characterized by the monosomy of an X chromosome. Subjects with this syndrome have in fact a cariotype 45 , $X$. It was first described by Ulrich and Turner in 1938 [15]. A pathognomonic element is ovarian dyskinesia with infertility as well as the cubitus valgus (the increase in the deviation of the forearm on the arm in the direction of the fully extended limb radio). Low stature and associated malformations such as wide chest (shield), reduction of the fourth metacarpal bone, peripheral lymphoedema at birth, hypoplastic nails, multiple pigmented nevi are other indicative signs for the diagnosis [13, 14]. The main cause is the nondisjunction of the sexual chromosome during meosis, typically due to an error in spermatogenesis, that causes the loss of the paternal sexual chromosome. Prenatal diagnosis can be made with amniocentesis and chorionic villus sampling. The incidence is estimated to be 1 in 2500 live- born females [13].

The abnormal ovarian function is responsible for alterations in hormonal secretion such as androgens, thyroid hormones and in particular $\mathrm{GH}$, the growth hormone. Considering the fact that, as we have said, these patients have short stature, they will need the hormone GH to reach normal height, and it will have to be administered before the age of $6[13]$. On the contrary, estrogen therapy is used at puberty [15]. The lack of an $\mathrm{X}$ chromosome also affects the size and shape of some craniofacial structures. In particular the jaw and mandible are short and retrognatic [13]. Besides, since at the level of the short arm (locus p22) of the $\mathrm{X}$ chromosome there are genes that are involved in the modulation of odontogenesis such as the AMGX gene that encodes amelogenin, a protein secreted by ameloblasts, in these patients there may be alterations in the production of enamel both quantitatively, and consequently with small size of deciduous and permanent teeth, qualitatively with enamel hypoplasia and therefore an increased risk of tooth decay [16]. In these patients, a tendency to the second skeletal class, a reduction in the posterior facial height and the lengthening of the lower third of the face were also observed. It was also possible, through clinical and radiographic investigations, to identify an ogival palate with cross-sectional incompetence between the arches. Dental abnormalities include tooth size, number and shape of the crown, reduced in a mesiodistal sense, and roots that have a more complex structure especially at the premolar level [14].

Wedge-shaped incisors, the altered shape, the volume and the small number of the cusps, or the triangular molar occluded surface are other found anomalies.

Precisely because the patient with Turner syndrome is at high risk of dental caries, it will be the task of the dentist, once the diagnosis is confirmed, to inform the family about the importance of oral health and the need for interventions aimed at promoting oral hygiene such as adequate nutrition, use of topical fluoride, periodic visits and, if necessary, sealings of grooves and cracks. Early diagnosis should be accompanied by treatment of dental abnormalities [3]. Orthodontic therapy can also be used to expand the palate, so that nasal breathing may improve. The precocity of this type of intervention is a guarantee of a better success of the therapy itself. In conclusion, the dentist, in his clinical practice, may have to deal with subjects suffering from such a syndrome whose oral manifestations may provide a first clue for the early diagnosis of the pathology [16].

\section{SAPHO SYNDROME}

SAPHO is an acronym that stands for synovitis, acne, pustulosis, hyperostosis, and osteitis.

These are the main symptoms of a rare condition, belonging to the class of reumatich diseases [17], introduced for the first time by Chamot et al., in 1987 [18].

Its prevalence, among Caucasians, has been estimated to be no greater than 1 case per 10,000 people, whereas in the Asiatic population it is even lower [19].

Considering the age of the onset, several studies agree on stating that this condition is more common in childhood and early adulthood [19, 20].

Moreover, it seems to be a predilection for the female sex, with a ratio between men and women of about $1: 2[21]$.

SAPHO is described as a disorder characterized by skin lesions, such as palmoplantar pustolosis (PPP), acne and psoriasis, and osteoarticular manifestations that mainly occur in clavicles, s ternum, sternoclavicular joints, sacroiliac region and spine [19].

The exact etiology still remains unclear, but it can be probably found in a combination of genetic, infectious and microbiological factors [22].

According to the results found in literature, temporomandibular involvement is clinically rare, and it is reported to be present in $10 \%$ of cases [18].

However, it's worth considering the presence of some radiological evidences that can lead the dentist to consider this syndrome. As a matter of fact, in some patients oral manifestations may precede the development of skin lesions, that can also be absent in $25 \%$ of cases [22]. 


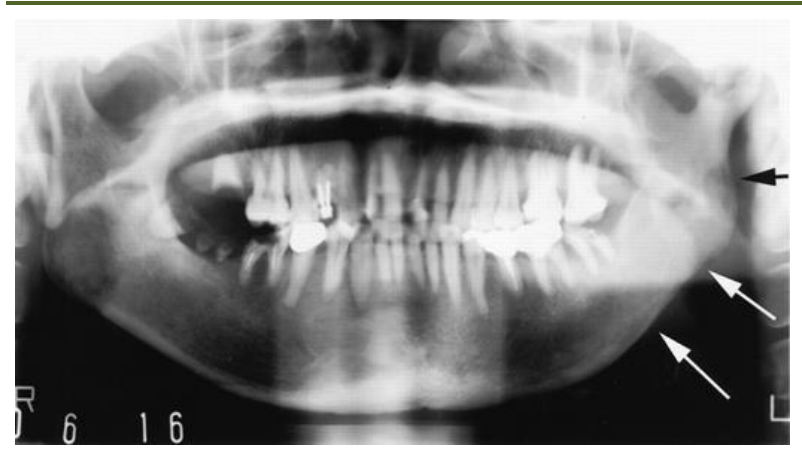

Fig-4: Panoramic radiograph of a patient affected by a longstanding SAPHO. In this image are shown osteolytic lesions (arrows) in the context of osteosclerotic lesions. Image courtesy of Yoshikazu Suei

Conforming to several studies 6 , the mandibular lesions typically consist in diffuse sclerosing osteomyelitis (DSO) in adults, and in chronic recurrent multifocal osteomyelitis (CRMO) in children and young adults.

As stated in the diagnostic criteria for SAPHO (from Kahn MF, 2003 ACR 67th Annual Scientific Meeting), in this syndrome the osteomyelitis is usually sterile (with the exception of some biopsy samples in which the Propionibacterium acnes was found).

The posterior body and the ramus seem to be the most affected part of the mandible [24]; quite the opposite, the temporomandibular joint is rarely involved.

More specifically, considering the lesions of the mandibular corpus, they usually are unilateral [19].

Regarding the radiological evidences of this syndrome, there are some findings that have been recognized to be widely diffused in people affected by SAPHO.

In particular, the panoramic radiograph shows periosteal reaction combined with sclerotic and lytic lesions (Fig-4), resorption of cortical and trabecular bone (Fig-5), and bone enlargement [19, 20, 23].

Besides, in the early stages of this syndrome, and also during the exacerbation periods, the osteolytic lesions were prevalent.

On the other side, with the progression of the disease they are partially replaced by osteosclerotic lesions, that are characteristic of the later stages [23].

Physical examination reveals that most patients affected by SAPHO complain of local inflammatory symptoms, pain, mandibular swelling and tenderness [17, 19-22, 24].

In addition, as shown by the case of a 26 -yearold woman, bilateral palatine tonsils and reactive lymphadenopathy may also be present [19].

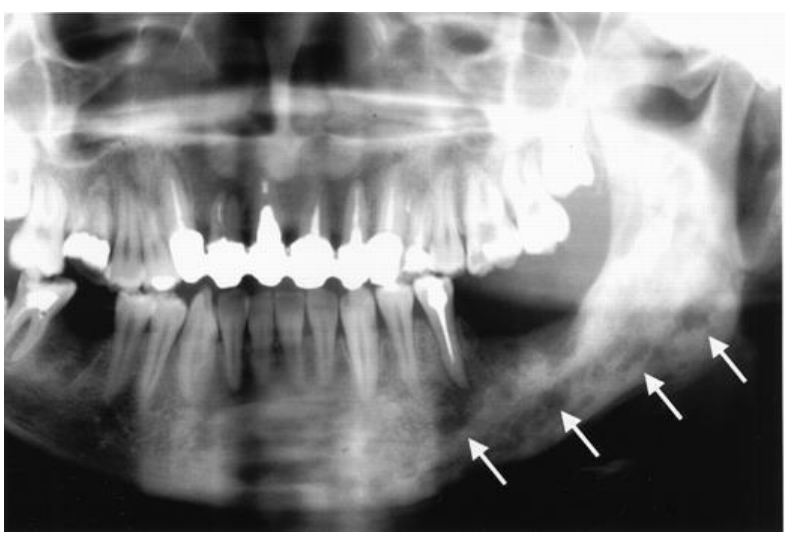

Fig-5: Panoramic radiograph of a patient affected by SAPHO. The arrows indicate the well visible bone resorption on the left mandible. Image courtesy of Yoshikazu Suei

Another clinical finding is represented by a variable restricted mouth opening, that is thought to be caused by a fusion of TMJ [20].

As a matter of fact, although it is uncommon, when the DSO spreads to the temporomandibular joint the patient may develop ankyloses [20-22] or severe destruction of the condyle [17].

In conclusion, when the mandibular manifestations occur without any other skin lesions, the diagnosis of SAPHO syndrome is quite difficult.

Nevertheless, if a patient has a mandibular osteitis of unknown etiology, and the orthopantomography reveals the above listed patterns, the diagnosis of SAPHO syndrome should be considered.

\section{NEUROFIBROM AT OSIS TYPE 1}

Neuro fibromatos is type 1 (NF1) is neurocutanous-skeletal syndrome caused by mutations in the NF1 tumor suppressor gene, whose location is $17 q 11.2$ [25].

This condition is one of the most frequently inherited genetic diseases and, as the matter of fact, its birth incidence is 1 case per 2000 [25].

In addition, also the prevalence is quite high, with 1 person affected per 2000-3000 people [25]. Considering the transmission, the inheritance follows an autosomal dominant trait [1], and the penetrance is complete [26].

According to several studies, there is no predilection for any gender or population [26]. The characteristic hall marks of neurofibromatosis type 1 are the so called café au lait spots, and the growth of a variable number of cutaneous and plexiform neurofibromas (described as benign tumors of the nerve sheath) $[25,27]$. 
More specifically, in the oral and maxillofacial region it is widely diffused the presence of facial plexiform neurofibromas (FPNs), which mainly develop from the three branches of the trigeminal nerve [26-28].

The connection between neurofibromatosis type 1 and different jaw and teeth alterations is demonstrated by various studies [26-29].

For example, Friedrich et al., [5] have examined the role of this syndrome in the development of supernumerary teeth (ST), analyzing panoramic radiographs of 200 people affected by neurofibromatosis type 1 (with or without FPNs).

The study revealed that 12 NF1 patients $(6 \%)$ had supernumerary teeth, which were distributed in 3 incisors, 8 premolars, and 12 molars.

In particular, they mainly developed in only one jaw (10 of 12 cases), that was represented by the upper jaw in seven patients, and by the mandible in three other patients.

In the last two cases, both jaws showed supernumerary teeth. On the other side, no ST were founded in the control group, composed of 200 people without evidence of any syndromic disease.

However, although the ST occurred in the region of facial plexiform neurofibromas, the numerical aberrations do not depend from the presence of the neoplasm itself.

In another study Friedrich et al., [26] analyzed 48 patients affected by NF1, distinguishing them in two groups; the first included people with facial plexiform neurofibromas, whereas in the second there were those with disseminated cutaneous neurofibromas.

They found that retained, or supernumerary, or more distally positioned teeth were quite exclusively represented in patients with FPNs. In the same study it was also suggested a correlation between FPNs and the aplasia of the second lower molar of the affected side.

As supposed by several authors [26], the tumorous growth may cause a suppression of development of the second molar germ.

Besides that, it is also worth focusing on other radiographic findings, that may suggest the presence of neurofibromatosis type 1 .

In a review published on the Orphanet Journal of Rare Diseases [25], Visnapuu et al., introduced a new NF1 related manifestation that was represented by the periapical cemental dysplasia (PCD).
Considering the radiological aspect of this condition, it may be described as a radiolucent periapical lesion in vital teeth. Curiously, none of the male patient in the study showed this kind of alteration.

Moreover, neurofibromatosis type 1 is also responsible for some other mandibular changes which can be detected in panoramic radiographs.

According to various studies [25], the most frequent findings are intrabony lesions, such as wide mandibular canals and enlarged mental foramina (Fig. 6), and different skeletal deformities [28].

A well visible alteration is for example represented by a change in the shape of the coronoid or condylar process, resulting in a narrowed bicortical diameter in the anterior- posterior direction.

In particular, due to the reduction of the osseous volume, the articular process becomes similar to a pencil (Fig-6). Condylar deformities are widely diffused in NF1 patients, as clearly highlighted by a study in which the half of the syndromic considered people showed this kind of change [28].

Another interesting result was obtained comparing people with only disseminated cutaneous neurofibromas, in the territories of the third trigeminal branch, and people with facial plexiform neurofibromas affecting the same branch.

The presence of an altered mandibular condyle was significantly more frequent in the second group.

Moreover, also the enlargement of the mandibular notch was mainly represented in patients with FPNs (Fig-6).

An additional deformity consists in a variable alteration of the mandibular angle of the FPNs' affected side, which may be completely missing or strongly reduced until it becomes, in some cases, a spine-like extension.

Once again, the association of these findings and FPNs affecting the third trigeminal branch, rather than the first or the second, is significant. As the matter of fact, patients affected only in the ophthalmic or in the maxillary branch showed a lower number of this kind of deformity.

In the same study, the half of the FPNs group had a shape alteration of the glenoid fossa (Fig. 6), whereas only one case was registered among people affected by disseminated cutaneous neurofibromas.

The changes that may occur in this region are quite different, but they are mainly described as a widening and a flattering of the fossa. Sometimes, it 
may also be a conspicuous gap between the articular process and the glenoid fossa, which can be so wide that there is no longer any understandable anatomical relationship between the two structures.

In conclusion, a single finding of those described is not specific for neurofibromatosis type 1 [28].

However, the simultaneous presence of some of them, in addition to other signs, such as an optic nerve glioma and at least six café au lait spots, may be highly suggestive of this syndrome.

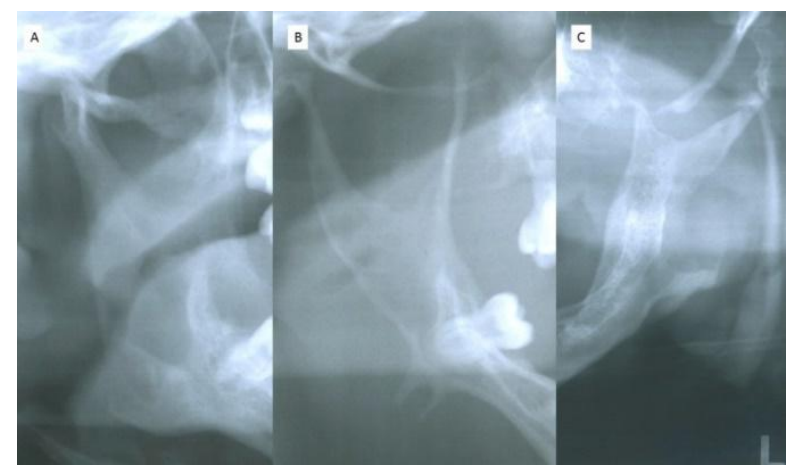

Fig-6: Orthopantomograms of three patients with a FPN affecting the third branch of the trigeminal nerve

In these images are clearly represented the mandibular alterations linked to neurofibromatosis type 1 , such as the deformation of glenoid fossa, the pencillike articular process, the deep notch and the widened mandibular foramen. Images courtesy of Reinhard E. Friedrich.

\section{WILLIAMS SYNDROME}

Williams syndrome is also known as WilliamsBeuren syndrome (WBS), by its discoverers Dr. J. C. P. Williams and Dr. A. J. Beuren [30]. It is a rare genetic disorder with a prevalence of 1 in 7500 newborns [31], but the incidence could be higher due to underdiagnosis [30]. It can affect both sexes and all ethnic groups without distinction. This pathology originates from a microdeletion in the region q.11.23 on the long arm of chromosome 7 and determines the loss of 26 to 28 contiguous genes, including elastin, that is an essential component of connective tissues [32]. Normally it arises sporadically at birth or in early childhood, but hereditary cases with a dominant autosomal transmission are also reported [31].

It is diagnosed through the FISH (Fluorescent in situ hybridization) technique, or through new techniques that are represented by qPCR (quantitative realtime polymerase chain reaction) and Array-CGH (microarray-based comparative genomic hybridization) that allow a more accurate diagnosis [32].

Several clinical features are described in patients with WBS; facial dysmorphisms, cardiological problems, overvalvular aortic $\mathrm{s}$ t enosis, mental retardation with a characteristic cognitive profile and congenital hypercalcemia [30-32]. Systemic comorbidities include hypertension, risk of sudden death, joint limitation and premature gray hair [32]. Facial dysmorphism is considered one of the main features regarding this pathology. Patients have a peculiar face called "elfin face" because of periorbital fat accumulation, wide forehead, flattened central face, small nose, thick lips, elongated philtrum, micrognatia (an incomplete development of the jaw that appears small) and large mouth. Within the oral cavity the main anomalies are malocclusion of I and III Angle's class sometimes accompanied by a raised palatine arch, deep open bites and crossed anterior bite. Specifically, the dental anomalies concern: generalized diastemas, hypodontia, microdontia, short dental roots, molten teeth, enamel hypoplasia (especially on the first permanent molars), agenesis and crown shape anomalies of some dental elements concerning the upper lateral incisors that are screwdriver-shaped, and taurodontism of the first and second lower molar is observed [30-32].

Talita Castro et al., evaluated 52 subjects affected by this syndrome both from a clinical and radiographic point of view, also performing ananalys is of the temporomandibular joint functioning, an assessment of the risk of caries through DMFT/dmft and periodontal disease through bleeding on probing, CPI (community periodontal record) and SPR (simplified periodontal record). Almost all subjects (51 out of 52) presented at least one anomaly in dental development, the most common of which were diastemas, malocclusion and hypodontia. The presentation of diastemas was attributed to the small size of dental crowns. Clinically, in addition to generalized diastemas, melted teeth were also observed, a bilateral fusion between the lower lateral incisor and canine and a high percentage of both upper and lower screwdriver-shaped incisors were reported. OPT has been used as a support for the evaluation of microdontia of the upper and lower lateral incisors and hypodontia of the upper lateral incisors and premolars, and of the lower second premolars [32].

This was most likely the first study that evaluated temporomandibular joint movements in subjects with WBS. The results showed that some patients participating in the study had reduced movements and functional changes in TMJ, but were not statistically significant when compared to the general population.

DMFT was used to assess the risk of caries, which has been defined as low in these patients but it is in contradiction to what was reported by Carolina Paes Torres et al., in their study where a 7-year-old Caucasian child with WBS with a high risk of caries was followed. Also in this case an initial clinical 
evaluation was performed, which revealed a high interdental space, hypomineralization of incisor enamel, hypoplasia and carious lesions in the lower and upper first molars, followed by radiographic analysis that showed a normal dental rash associated with age and agenesis of the right lower second premolar. The presence of the erupted deciduous tooth in the oral cavity can in fact be noted on the OPT but the lack of the dental germ of the permanent tooth is observed [30]. Shirlene-Barbosa-Pimentel Ferreira et al., also observed agenesis of the second lower and upper premolar, and of the lower lateral incisors in some of the patients followed. Also in this study malocclusion is the main manifestation, accompanied by high palatine arch that for the first time has been described thanks to these authors [31].

Patients must then undergo an X-ray analysis in support of a clinical evaluation that allows the detection of anomalies typical of the disease and helps the dentist to better manage the patient.

As noted in the articles, patients should undergo dental prophylaxis before the treatment and they have to be trained in oral hygiene to reduce the risk of caries, because the development of carious lesions can lead to serious complications such as subacute bacterial endocarditis.

\section{SCHIMKE IMMUNO}

Schimke's immuno-osseous dysplasia (SIOD), or spondyloepiphyseal dysplasia, first described in 1971 , is a rare autosomal recessive disease with an incidence of 1caseper 1,000,000-3,000,000 newborns [33]. It is caused by a bi-allelic mutation of SAMRCAL1 on chromosome 2 coding for a protein belonging to the $\mathrm{SnF} 2$ protein family involved in chromatin remodeling [34]. Subjects with a severe disease die within the first decade of life, while if the disease is in a mild form patients reach adult life. The main features of this pathology are spondylopophyseal dysplasia, renal dysfunction, T- cell immunodeficiency, protuberant trunk, short limbs and facial dysmorphism [34-36]. The presence of vascular abnormalities causing significant morbidity and mortality in these patients have also been observed [35].

The face has a triangular shape, with a wide nasal bridge and bulbous nose tip, small eyelid fissures, hyperpigmented macules and long upper lip. The neck is short and the hairline is low [35].

Not much is known about oral manifestations, but as reported by $M$. Morimoto et al. and later supported by M. Gendronneau et al., 66\% of SIOD patients have oral manifestations, some of which are specific to this disease. Microdontia, hypodontia, molar roots' hypoplasia, bulbous molar crowns and radiopacity of deciduous and permanent molars often occur. A typical manifestation, that we can see through radiographic investigation, is the presence of a disproportion between the crowns and the molar roots, which can also be severe in some cases, with a marked cervical constriction. Precisely because of this malformation, molar and even premolar teeth, both deciduous and permanent, are defined as bell-shaped or tulip-shaped, while anterior teeth have a normal shape $[34,36]$. Because of the exclusive involvement of posterior teeth, M. Morimoto et al., initially hypothesized that SMARCAL1 was not expressed in the anterior teeth, but later, through analysis of died patients, the presence of SMARCAL1 was also confirmed in the anterior teeth. Then, in the same study, information on the expression of SMARCAL1 (highly expressed in the developing human tooth) in the external and internal dental epithelium and in the enamel nodes is reported. The molars have a more complex development and so they require secondary and tertiary enamel nodes, for this reason they show these alterations [36].

To explain the nature of the pathology, a culture examination, which was reported in the study above mentioned, was performed on skin fibroblasts affected by SIOD. The examination showed that SMARCAL 1 deficiency significantly modifies the gene responses of WNT3A, BMP4 and TGFb1.

WNT3A regulates tooth number, size and shape, BMP4 mediates epithelial-mesenchymal interactions, regulates enamel node formation and Hertwig sheath formation, regulates tooth number, size and shape, while TGFb1 also modulates fibroblast differentiation. This model, however, needs further studies [36].

Diagnosis often takes a long time, but the presence of uncommon dental abnormalities are helpful in making an early diagnosis. Clinically, patients with SIOD may present grey and yellow teeth as reported by Marcio A. da Fonseca and M. Gendronneau et al., in their studies $[34,35]$. In their article M. Gendronneau et al., visited and followed two children, the younger one reported microdontia with large interdental spaces in addition to discoloration. The second child and the sixyear-old child described in Marcio A. da Fonseca's article showed teeth of normal size.

The two children with SIOD followed by $\mathrm{M}$. Gendronneau et al., underwent dental follow up every 6 months and a treatment with fluoridated toothpastes; the children and their parents were also taught the importance of oral hygiene with proper nutrition. 


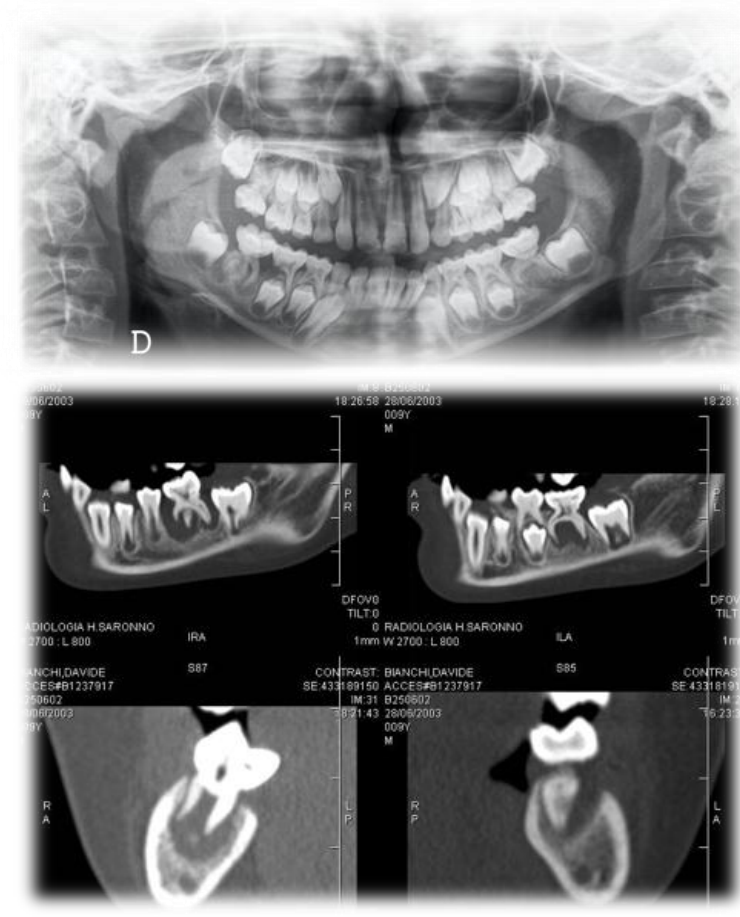

Fig-7: OPT and TAC of about 8-year-old child affected by Schimke Immuno. It is observed the cervical constriction in premolars and molars. They have a tulip shape, typical characteristic of the disease

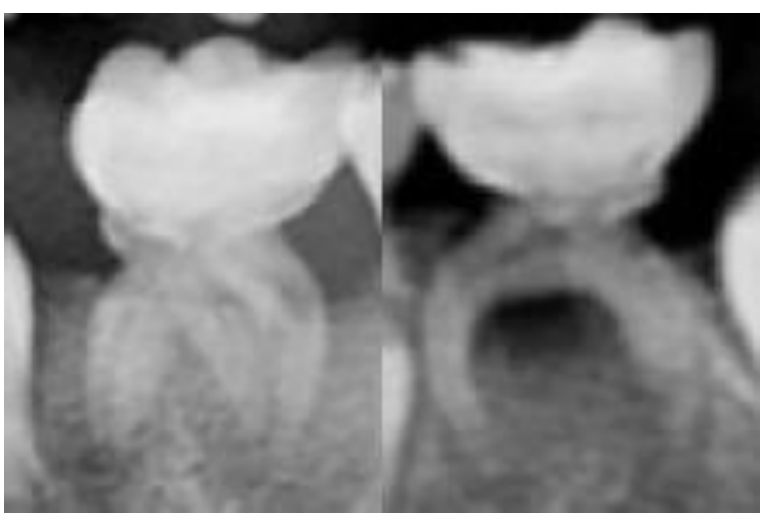

Fig-8: Details of the typical tulip shape of the molars.

After the clinical evaluation of the children, in both studies, an OPT was performed and it showed a dental eruption appropriate to their age, and it detected the typical characteristics of the disease: bulbous crowns with marked cervical constriction of both deciduous and permanent molars and premolars, which give the typical bell or tulip appearance, reduced or absent pulpitary chambers, short and thin roots and marked radiopacity of the crowns.

M. Gendronneau et al., through OPT observed in the younger child another particular sign that is the agenesis of permanent second premolars, a characteristic previously reported by $\mathrm{M}$. Morimoto et al. as one of the frequent manifestations of the disease [3436].
The dental phenotype in SIOD is similar to dentinogenesis imperfecta (DI) type II, a primary collagen defect common to both deciduous and permanent bones and teeth as reported by Marcio A. da Fonseca[35]. The author described previous studies in which it was hypothesized that the association of abnormal teeth and spondyloepiphyseal dysplasia were linked to a generalized disturbance of the connective tissue matrix and for these reasons he performed an initial temporary diagnosis of DI in the child followed. In DI the teeth have a discoloration, and enamel fractures, tooth wear, short blunt roots, bulbous crowns, obliterated pulp chambers, soft dentine and enamel hypoplasia often occur. Although some characteristics are common, M. Morimoto et al. state that teeth of SIOD subjects rarely show discoloration, enamel hypoplasia and soft dentine and that the SIOD dental phenotype is unique [36].

In conclusion, SIOD is a systemic disease with unique oral manifestations that allow, if correctly identified, to reduce the time of diagnosis and make the diagnosis less complicated. Doctors and dentists must cooperate both in the diagnosis and in the treatment of patients, for a complete management of the patient.

\section{CONCLUSION}

Rare diseases considered in this narrative review have both systemic and oral manifestations. An early diagnosis is difficult to make; hence the cooperation of several specialists is essential.

Among these, the dentist, with the support of radiographic instruments such as the OPT, plays a key role.

As a matter of fact, in these syndromes the timely detection of clinical and radiographic alterations could raise a diagnostic suspicion that may lead the dentist to refer the patient to other specialists for further investigations. However, considering the oral manifestations related to the analyzed diseases, periodic dental follow-ups remain of fundamental importance in order to monitor any development.

\section{REFERENCES}

1. Wagel J, Łuczak K, Hendrich B, Guziński M, Sąsiadek M. Clinical and radiological features of nonfamilial cherubism: a case report. Polish Journal of Radiology. 2012 Jul;77(3):53-57.

2. Redfors M, Jensen JL, Storhaug K, Prescott T, Larheim TA. Cherubism: panoramic and CT features in adults. Dentomaxillofacial Radiology. 2013 Dec;42(10):20130034.

3. Lima GD, Almeida JD, Cabral LA. Cherubism: clinicoradiographic features and treatment. Journal of oral \& maxillofacial research. 2010 Apr;1(2):e2.

4. Silva EC, Silva GC, Vieira TC. Cherubism: clinicoradiographic features, treatment, and long- 
term follow-up of 8 cases. Journal of oral and maxillofacial surgery. 2007 Mar 1;65(3):517-22.

5. Papadaki ME, Lietman SA, Levine MA, Olsen BR, Kaban LB, Reichenberger EJ. Cherubism: best clinical practice. InOrphanet journal of rare diseases 2012 Jun 1 (Vol. 7, No. S1, p. S6). BioMed Central.

6. Gorlin RJ, Goltz RW. Multiple nevoid basal-cell epithelioma, jaw cysts and bifid rib: a syndrome. New England Journal of Medicine. 1960 May 5;262(18):908-12.

7. Spiker AM, Troxell T, Ramsey ML. Gorlin Syndrome (Basal Cell Nevus). In StatPearls. StatPearls Publishing. 2020.

8. Pino LC, Balassiano LK, Sessim M, de Almeida AP, Empinotti VD, Semenovitch I, Treu C, Lupi O. Basal cell nevus syndrome: clinical and molecular review and case report. International journal of dermatology. 2016 Apr;55(4):367-75.

9. Muzio LL. Nevoid basal cell carcinoma syndrome (Gorlin syndrome). Orphanet journal of rare diseases. 2008 Dec 1;3(1):32.

10. MacDonald-Jankowski DS. Keratocystic odontogenic tumour: systematic review. Dentomaxillofacial Radiology. 2011 Jan;40(1):123.

11. Thomas N, Vinod SV, George A, Varghese A. Gorlin-Goltz syndrome: An often missed diagnosis. Annals of maxillofacial surgery. 2016 Jan;6(1):120-124.

12. Lazaridou MN, Dimitrakopoulos I, Tilaveridis I, Iliopoulos C, Heva A. Basal cell carcinoma arising in association with a maxillary keratocyst in a patient with Gorlin-Goltz syndrome. Report of a case. Oral and maxillofacial surgery. 2012 Mar 1;16(1):127-31.

13. Ahiko N, Baba Y, Tsuji M, Horikawa R, Moriyama K. Investigation of maxillofacial morphology and oral characteristics with Turner syndrome and early mixed dentition. Congenital anomalies. 2019 Jan;59(1):11-7.

14. Kusiak A, Sadlak- Nowicka J, Limon J, Kochańska B. Root morphology of mandibular premolars in 40 patients with Turner syndrome. International Endodontic Journal. 2005 Nov;38(11):822-6.

15. López ME, Bazán C, Lorca IA, Chervonagura A. Oral and clinical characteristics of a group of patients with Turner syndrome. Oral Surgery, Oral Medicine, Oral Pathology, Oral Radiology, and Endodontology. 2002 Aug 1;94(2):196-204.

16. Szilágyi A, Keszthelyi G, Nagy G, Madléna M. Oral manifestations of patients with Turner syndrome. Oral Surgery, Oral Medicine, Oral Pathology, Oral Radiology, and Endodontology. 2000 May 1;89(5):577-84.

17. Kodama Y, Tanaka R, Kurokawa A, Ohnuki H, Sultana S, Hayashi T, Iizuka T, Takagi R. Severe destruction of the temporomandibular joint with complete resorption of the condyle associated with synovitis, acne, pustulosis, hyperostosis, and osteitis syndrome. Oral Surgery, Oral Medicine, Oral Pathology and Oral Radiology. 2013 Aug $1 ; 116(2):$ e128-33.

18. Chamot AM, Benhamou CL, Kahn MF, Beraneck L, Kaplan G, Prost A. Acne-pustulosishyperostosis-osteitis syndrome. Results of a national survey. 85 cases. Revue du rhumatisme et des maladies ostéo-articulaires. 1987 Mar 1;54(3):187-96.

19. Kikuchi T, Fujii H, Fujita A, Sugiyama T, Sugimoto H. Mandibular osteitis leading to the diagnosis of SAPHO syndrome. Case reports in radiology. 2018 Jun 13;2018.

20. Utumi ER, Sales MA, Shinohara EH, Takahashi A, Coracin FL, Rocha RG, Cavalcanti MG. SAPHO syndrome with temporomandibular joint ankylosis: clinical, radiological, histopathological, and therapeutical correlations. Oral Surgery, Oral Medicine, Oral Pathology, Oral Radiology, and Endodontology. 2008 Mar 1;105(3):e67-72.

21. Mueller-Richter UD, Roldán JC, Mörtl M, Behr M, Reichert TE, Driemel O. SAPHO syndrome with ankylosis of the temporomandibular joint. International journal of oral and maxillofacial surgery. 2009 Dec 1;38(12):1335-41.

22. McPhillips A, Wolford LM, Rodrigues DB. SAPHO syndrome with TMJ involvement: review of the literature and case presentation. International journal of oral and maxillofacial surgery. 2010 Dec 1;39(12):1160-7.

23. Suei Y, Taguchi A, Tanimoto K. Diagnostic points and possible origin of osteomyelitis in synovitis, acne, pustulosis, hyperostosis and osteitis (SAPHO) syndrome: a radiographic study of 77 mandibular osteomyelitis cases. Rheumatology. 2003 Nov 1;42(11):1398-403.

24. Zemann W, Pau M, Feichtinger M, Ferra-Matschy B, Kaercher H. SAPHO syndrome with affection of the mandible: diagnosis, treatment, and review of literature. Oral Surgery, Oral Medicine, Oral Pathology, Oral Radiology, and Endodontology. 2011 Feb 1;111(2):190-5.

25. Visnapuu V, Peltonen S, Alivuotila L, Happonen RP, Peltonen J. Craniofacial and oral alterations in patients with Neurofibromatosis 1. Orphanet journal of rare diseases. 2018 Dec 1;13(1):131.

26. Friedrich RE, Giese M, Schmelzle R, Mautner VF, Scheuer HA. Jaw malformations plus displacement and numerical aberrations of teeth in neurofibromatosis type 1: a descriptive analysis of 48 patients based on panoramic radiographs and oral findings. Journal of Cranio-Maxillofacial Surgery. 2003 Feb 1;31(1):1-9.

27. Friedrich RE, Reul A. Supernumerary molars and wisdom tooth shape alterations in patients with neurofibromatosis type 1 . Journal of oral \& maxillofacial research. 2017 Oct;8(4):e5.

28. Friedrich RE, Reul A. A combination of skeletal deformations of the dorsal mandible and 
temporomandibular region detected in orthopantomograms of patients with neurofibromatosis type 1 indicates an associated ipsilateral plexiform neurofibroma. Journal of Cranio-Maxillofacial Surgery. 2018 Jul 1;46(7):1091-104.

29. Friedrich RE, Scheuer HT, Kersten JF, Scheuer HA. Supernumerary teeth of permanent dentition in patients with neurofibromatosis type 1. Journal of Cranio-Maxillofacial Surgery. 2020 Jan 1;48(1):98-104.

30. Torres CP, Valadares G, Martins MI, Borsatto MC, Díaz-Serrano KV, Queiroz AM. Oral findings and dental treatment in a child with WilliamsBeuren syndrome. Brazilian Dental Journal. 2015 Jun;26(3):312-6.

31. Shirlene-Barbosa-Pimentel Ferreira MM, Viana NG, Letícia-Lima Leão RA, Ricardo-Della Coletta MJ, de Aguiar HM. Oral findings in WilliamsBeuren syndrome. Medicina oral, patologia oral y cirugia bucal. 2018 Jan;23(1):e1-e6.

32. Castro T, de Paula Martins Santos C, de Oliveira Lira Ortega A, Gallottini M. Oral characteristics and medical considerations in the dental treatment of individuals with Williams syndrome. Special Care in Dentistry. 2019 Mar;39(2):108-13.

33. Santangelo L, Gigante M, Netti GS, Diella S, Puteo F, Carbone V, Grandaliano G, Giordano M, Gesualdo L. A novel SMARCAL1 mutation associated with a mild phenotype of Schimke immuno-osseous dysplasia (SIOD). BMC nephrology. 2014 Dec;15(1):1-5.

34. Gendronneau M, Kérourédan O, Taque S, Sixou JL, Bonnaure-Mallet M. Dental abnormalities and preventive oral care in Schimke immuno-osseous dysplasia. European Archives of Paediatric Dentistry. 2014 Jun 1;15(3):217-21.

35. da Fonseca MA. Dental findings in the Schimke immuno- osseous dysplasia. American journal of medical genetics. 2000 Jul 17;93(2):158-60.

36. Morimoto $\mathrm{M}$, Kerouredan $\mathrm{O}$, Gendronneau $\mathrm{M}$, Shuen C, Baradaran-Heravi A, Asakura Y, Basiratnia M, Bogdanović R, Bonneau D, Buck A, Charrow J. Dental abnormalities in Schimke immuno-osseous dysplasia. Journal of dental research. 2012 Jul;91(7_suppl):S29-37. 